\title{
LA PARTICIPACIÓN DE LOS ESTUDIANTES EN EL PROCESO DE EVALUACIÓN: UNA EXPERIENCIA EN EL AULA UNIVERSITARIA
}

The participation of students in the evaluation process: an experience in the university classroom

A participação dos alunos no processo de avaliação: uma experiência numa sala de aula universitária

\section{Miriam Sonlleva Velasco (1)}

(1) Universidad de Valladolid, España. Teléfono: +34 921112294. miriam.sonlleva@uva.es

\section{Resumen}

La experiencia que presentamos trata de mostrar cómo se ha llevado a cabo un sistema de evaluación formativa en la asignatura de Educación para la Paz y la Igualdad, del Programa de Estudios Conjunto de Grado en Educación Infantil y Primaria de la Facultad de Educación de Segovia. Dos rasgos caracterizan esta experiencia: la participación del alumnado en el sistema de evaluación y la labor del docente como guía y acompañante del aprendizaje. Los resultados no solo muestran cómo la puesta en práctica de sistemas de evaluación formativa en el nivel universitario repercute de forma positiva en las calificaciones de los estudiantes, sino también la incidencia de esta forma de evaluar en la adquisición de competencias por parte de los docentes en formación y en su formación en los contenidos de la materia.

Palabras clave: Enseñanzas universitarias; Evaluación Formativa; Formación Inicial del Profesorado; Participación del alumnado

\footnotetext{
Abstract

The experience that we present tries to expose how a formative assessment system has been carried out in the subject of Education for Peace and Equality, of the Doble Grade in Early Chilhood Education and Primary Education in the Faculty of Education of Segovia. Two features characterize this experience: the participation of students in the evaluation system and the work of the teacher as a guide and accompaniment to learning. The results expuse how the implementation of formative assessment systems at university level has a positive impact on the students' grades and the impact of this form of

La participación de los estudiantes en el proceso de evaluación: una experiencia en el aula universitaria 
evaluation on the acquisition of competences by teachers in training. We also appreciate an improvement in the training of students related to the contents of the subject Keywords: University education; Formative assessment; Initial Teacher Training; Participation of students

\section{Resumo}

A experiência que apresentamos tenta mostrar como tem sido realizado um sistema de avaliação formativa na disciplina de Educação para a Paz e Igualdade do Programa de Estudos Conjuntos da Licenciatura em Educação Pré-Escolar e Primária da Faculdade de Educação de Segóvia. Duas características caracterizam esta experiência: a participação dos alunos no sistema de avaliação e o trabalho do professor como guia e acompanhante da aprendizagem. Os resultados não só mostram que a implementação de sistemas de avaliação formativa no ensino universitário tem um efeito positivo sobre as classificações dos alunos, mas também a incidência desta forma de avaliação na aquisição de competências por parte dos professores em formação e na sua formação nos conteúdos da disciplina.

Palavras-chave: Ensino universitária; Avaliação formativa; Formação inicial de professores; Participação de estudantes.

\section{Introducción}

El Espacio Europeo de Educación Superior viene reclamando desde comienzos de siglo un cambio en los procesos de enseñanza-aprendizaje que se llevan a cabo en las universidades. Dentro de estos procesos se encuentra la evaluación, a pesar de que su práctica sigue teniendo en nuestros días tintes tradicionales.

En línea con la idea de López-Pastor $(1999,2012,2017)$ defendemos en este nivel la necesidad de llevar a cabo un sistema de evaluación formativa y compartida que sea capaz de: a) mejorar los procesos de enseñanza-aprendizaje; b) permitir que el estudiante mejore sus conocimientos y capacidades; c) perfeccionar la competencia docente del profesor que imparte sus enseñanzas; y d) entender la evaluación desde una mirada pedagógica y humanizadora y no como mera calificación de lo aprendido.

Para poder cumplir con tales fines resulta necesario modificar la práctica docente, planteando tanto tareas de evaluación como tareas de aprendizaje, haciendo partícipes a

La participación de los estudiantes en el proceso de evaluación: una experiencia en el aula universitaria 
los estudiantes en la evaluación y ofreciendo una retroalimentación de los resultados obtenidos (Álvarez, 2008).

Siguiendo con estas directrices, el objetivo de nuestro trabajo es presentar los resultados de una experiencia de evaluación formativa aplicada en la Facultad de Educación de Segovia en el curso 2018/2019.

\section{Contextualización}

Nuestra experiencia se contextualiza en un grupo de 42 estudiantes matriculados en la asignatura de Educación para la Paz y la Igualdad, que se imparte en el primer curso del Programa de Estudios Conjunto de Grado en Educación Infantil y Primaria. La asignatura tiene una carga de 6 créditos ECTS y se encuentra en el módulo de formación básica de la titulación.

\section{Diseño y desarrollo}

El sistema de evaluación de la asignatura cuenta con tres vías (Tabla 1). Cada estudiante puede elegir al comienzo del cuatrimestre cuál seguir, asumiendo las responsabilidades que implica dicha elección.

Tabla 1.

Vias de evaluación ofrecidas en la asignatura Educación para la Paz y la Igualdad

\begin{tabular}{|c|c|c|}
\hline Sistema de evaluación & Requisitos & $\begin{array}{l}\text { Actividades de } \\
\text { aprendizaje }\end{array}$ \\
\hline Continua & $\begin{array}{l}\text { Participar en más del } 90 \% \text { de las } \\
\text { clases, entregar todas las actividades } \\
\text { de aprendizaje en tiempo y forma y } \\
\text { obtener al menos un } 4 \text { en la prueba } \\
\text { final. }\end{array}$ & $\begin{array}{l}\text { Portafolio de actividades } \\
\text { y reflexiones, PAT y } \\
\text { examen final. }\end{array}$ \\
\hline Mixta & $\begin{array}{l}\text { Realizar y superar tanto el PAT como } \\
\text { las actividades de aprendizaje. } \\
\text { Obtener al menos un } 4 \text { en la prueba } \\
\text { final. }\end{array}$ & $\begin{array}{l}\text { Portafolio de actividades, } \\
\text { PAT y examen final. }\end{array}$ \\
\hline Final & $\begin{array}{l}\text { Realizar y superar la prueba final con } \\
\text { una calificación igual o superior a } 5 \\
\text { puntos. }\end{array}$ & Examen final \\
\hline
\end{tabular}

Fuente: elaboración propia

Dado que más del 95\% de los estudiantes decidieron elegir la evaluación continua, expondremos los resultados obtenidos en esta vía. Como se muestra en la tabla 1, para el sistema de evaluación continua se trabaja con tres instrumentos: un portafolio o dossier de actividades; un Proyecto de Aprendizaje Tutorado (PAT), en el que, por grupos, los

La participación de los estudiantes en el proceso de evaluación: una experiencia en el aula universitaria 
estudiantes deben realizar la programación de un taller sobre uno de los contenidos de la asignatura y ponerlo en práctica en un colegio; y una prueba escrita. En estos instrumentos, el alumnado tiene una participación directa en su proceso de evaluación, como pasamos a detallar.

La primera actuación que se lleva a cabo en el aula después de la presentación de la asignatura y la lectura de la guía docente es el consenso entre docentes y estudiantes sobre el porcentaje de calificación de cada uno de los tres instrumentos. En esta asignatura se decidió para el curso 2018/ 2019 que el portafolio tuviera un peso de un 35\% de la nota final; el PAT un 40\%; y el examen un $25 \%$.

Cada una de las cuatro prácticas que componen el portafolio de la asignatura son el resultado del debate en clase de sus contenidos a través de textos y tertulias pedagógicas. Al ser entregadas en el plazo pactado reciben feedback y pueden ser modificadas en dos semanas por parte del estudiante para la mejora de su aprendizaje y de la calificación. Con relación al PAT, durante la elaboración escrita de la propuesta, nos reunimos con los estudiantes, debatimos cada punto redactado de la programación y hacemos juntos propuestas de mejora. Además, antes de llevar el PAT al centro hacemos una sesión de coevaluación con el objetivo de que el alumnado encuentre los puntos fuertes y débiles de cada programación. Durante su desarrollo en el aula, cada grupo es observado por otros compañeros y recogen su actuación en una ficha de observación. Al finalizar el PAT, de forma individual, se presenta un cuestionario de autoevaluación.

En relación al examen, realizamos una rúbrica con los propios estudiantes sobre los criterios generales a evaluar en cada pregunta. Una vez terminada la prueba y revisadas las respuestas ofrecidas por el alumnado, detallamos las puntuaciones ajustadas con sus propias respuestas. Además, mostramos la rúbrica y comentamos cada pregunta con el grupo, de tal forma que los estudiantes sean conscientes de su proceso de aprendizaje.

\section{Evaluación}

El análisis de los resultados obtenidos en la asignatura por los estudiantes muestra que este sistema de evaluación ha sido satisfactorio no solo en relación a la calificación (la media de clase supera los 7,8 puntos), sino también al aprendizaje. Las producciones de los alumnos demuestran cómo se ha producido una mejora de sus conocimientos en los temas relacionados con la asignatura y también en sus competencias como docentes en formación.

La participación de los estudiantes en el proceso de evaluación: una experiencia en el aula universitaria 
No queremos dejar de señalar que uno de los aspectos que han sido clave en estos resultados positivos ha sido asumir nuestro papel de guías y acompañantes en el proceso de enseñanza-aprendizaje. Además, el feedback ofrecido a lo largo del desarrollo de la asignatura, en correspondencia con trabajos como los de Nicol y Macfarlane (2006), nos ha ayudado a: mejorar la comunicación con nuestros estudiantes; facilitar la reflexión sobre su propio aprendizaje; promover un cambio en sus formas de trabajo; y ofrecer una formación individualizada, capaz de atender a sus necesidades.

\section{Conclusiones}

Como conclusiones de esta experiencia podemos destacar: a) la importancia de plantear en la formación universitaria sistemas de evaluación formativa que impliquen directamente al alumnado y repercutan en la mejora de sus competencias profesionales; y b) la relevancia del papel del docente en la evaluación como guía del aprendizaje y no como mero estimador de resultados.

Actualmente se está realizando la transferencia de este modelo de evaluación participativo a otros grupos de la Facultad de Educación de Segovia, lo que nos permitirá plantear futuras líneas de desarrollo al establecer comparaciones entre los resultados obtenidos en distintas asignaturas.

\section{Referencias}

Álvarez, I. (2008). Evaluación del aprendizaje: una mirada retrospectiva y prospectiva desde la divulgación científica. Revista Electrónica de Investigación Psicoeducativa, 14(1), 235-272.

López-Pastor, V.M (1999). Prácticas de evaluación en Educación Física: estudio de casos en Primaria, Secundaria y Formación del Profesorado. Valladolid: Universidad de Valladolid.

López-Pastor, V. M. (2012). Evaluación Formativa y Compartida en la Universidad: clarificación de conceptos y propuestas de intervención desde la Red Interuniversitaria de Evaluación Formativa. Psychology, Society \& Educatión, 4(1), 113-126.

López-Pastor, V.M. (2017). Evaluación Formativa y Compartida: evaluar para aprender y la implicación del alumnado en los procesos de evaluación y aprendizaje. En V.M López Pastor y A. Pérez Pueyo (Coords.). Evaluación Formativa y

La participación de los estudiantes en el proceso de evaluación: una experiencia en el aula universitaria 
Compartida en Educación: experiencias de éxito en todas las etapas educativas (pp. 34-68). León: Grupo IFAHE (Universidad de León).

Nicol, D., \& Macfarlane Dick, D. (2006). Formative assessment and self-regulated learning: a model and seven principles of good feedback practice. Studies in Higher Education, 31(2), 199-218.

La participación de los estudiantes en el proceso de evaluación: una experiencia en el aula universitaria 